\title{
Ultrafast time-domain spectroscopy based on high-speed asynchronous optical sampling
}

\author{
A. Bartels, ${ }^{\text {a) }}$ R. Cerna, C. Kistner, A. Thoma, F. Hudert, C. Janke, and T. Dekorsy \\ Department of Physics and Center for Applied Photonics, University of Konstanz, \\ D-78457 Konstanz, Germany
}

\begin{abstract}
High-speed asynchronous optical sampling (ASOPS) is a novel technique for ultrafast time-domain spectroscopy (TDS). It employs two mode-locked femtosecond oscillators operating at a fixed repetition frequency difference as sources of pump and probe pulses. We present a system where the $1 \mathrm{GHz}$ pulse repetition frequencies of two Ti:sapphire oscillators are linked at an offset of $\Delta f_{R}$ $=10 \mathrm{kHz}$. As a result, their relative time delay is repetitively ramped from zero to $1 \mathrm{~ns}$ within a scan time of $100 \mu \mathrm{s}$. Mechanical delay scanners common to conventional TDS systems are eliminated, thus systematic errors due to beam pointing instabilities and spot size variations are avoided when long time delays are scanned. Owing to the multikilohertz scan-rate, high-speed ASOPS permits data acquisition speeds impossible with conventional schemes. Within only $1 \mathrm{~s}$ of data acquisition time, a signal resolution of $6 \times 10^{-7}$ is achieved for optical pump-probe spectroscopy over a time-delay window of $1 \mathrm{~ns}$. When applied to terahertz TDS, the same acquisition time yields high-resolution terahertz spectra with $37 \mathrm{~dB}$ signal-to-noise ratio under nitrogen purging of the spectrometer. Spectra with $57 \mathrm{~dB}$ are obtained within $2 \mathrm{~min}$. A new approach to perform the offset lock between the two femtosecond oscillators in a master-slave configuration using a frequency shifter at the third harmonic of the pulse repetition frequency is employed. This approach permits an unprecedented time-delay resolution of better than 160 fs. High-speed ASOPS provides the functionality of an all-optical oscilloscope with a bandwidth in excess of $3000 \mathrm{GHz}$ and with $1 \mathrm{GHz}$ frequency resolution.
\end{abstract}

\section{INTRODUCTION}

Experiments in the field of ultrafast time-domain spectroscopy most commonly employ trains of pump and probe pulses derived from a single mode-locked laser. One pulse train travels a variable distance and the signal, e.g., reflectivity changes of a sample after pulsed optical excitation, is recorded as a function of the resulting time delay versus the other pulse train. In most cases the time delay is adjusted with mirrors mounted onto a mechanical translation stage or a vibrating membrane. While translation stages allow for several hundred picoseconds delay, they do not permit rapid scanning. Vibrating membranes allow for higher scan rates in the range of $10-100 \mathrm{~Hz}$ but are limited to approximately 10-100 ps time delay. A severe disadvantage of translating mirrors in general is the fact that it is impossible to eliminate residual variations of the beam pointing and spot size on the sample as time delays of several hundred picoseconds are realized by moving a stage by tens of centimeters. These effects are greatly unfavorable when phenomena with long decay times are investigated over nanosecond time delays due to an unresolvable ambiguity between real sample dynamics and experimental artifacts. Furthermore, scan rates in the range of several kilohertz are desirable for the investigation of dynamic phenomena where successive data sets are to be acquired within just a few milliseconds (e.g., experiments in pulsed magnetic fields, reaction dynamics in biological samples, etc.) or for imaging applications in order to reduce the scan time per pixel. In addition, screening systems and possible security-related applications of terahertz (THz) TDS call for faster data acquisition than possible with mechanical delay scanners in order to permit a high throughput (e.g., readout of DNA sensors, ${ }^{1}$ detection of explosives, ${ }^{2}$ hazardous substances, or illicit drugs in mail or other opaquely packaged items).

Asynchronous optical sampling (ASOPS) has already been demonstrated in 1987 with mode-locked picosecond lasers at low repetition rates $(82 \mathrm{MHz})$. It is a technique that enables ultrafast time-domain spectroscopy without a mechanical delay line. ${ }^{3}$ Two mode-locked lasers are linked at a fixed repetition frequency difference $\Delta f_{R}$ and serve as pump and probe lasers, respectively. Their relative time delay is linearly ramped between zero and the inverse repetition frequency of the pump laser at a rate given by $\Delta f_{R}$. More recently, ASOPS experiments have been performed with $82 \mathrm{MHz}$ repetition rate femtosecond lasers at $\Delta f_{R}$ values in the range of $100 \mathrm{~Hz}$ or below. ${ }^{4,5}$ However, even though femtosecond pulses were employed, the demonstrated bandwidth was still well below $1 \mathrm{THz}$. Our high-speed implementation of ASOPS operates with mode-locked Ti:sapphire femtosecond oscillators with repetition frequencies close to $1 \mathrm{GHz}$ and an offset frequency of $\Delta f_{R}=10 \mathrm{kHz}$. Thus, our system scans a temporal measurement window of approximately $1 \mathrm{~ns}$ within $100 \mu$ s of scan time. High-repetition rates in the range of $1 \mathrm{GHz}$ are generally favorable for ASOPS because they permit a higher scan rate and a better time-delay resolution at a given optical detection and data acquisition band- 
width (practically accessible values are in the range of 100 $\mathrm{MHz}$ ). This has been pointed out in the first demonstration of a high-speed ASOPS system where the technique has been applied to the field of terahertz time-domain spectroscopy. ${ }^{1}$ In the experiments by Janke et al. ${ }^{1}$ the time-delay resolution had been limited to 2.5 ps due to limitations of the repetition frequency stabilization and the relatively low $1.8 \mathrm{MHz}$ bandwidth of the detection hardware. More recently, high-speed ASOPS has been demonstrated for optical pump-probe spectroscopy and for THz-TDS with a time delay resolution of 230 fs. 6,7

Here, we report a high-speed ASOPS system with significantly improved repetition frequency stabilization. Two mode-locked femtosecond oscillators are offset locked at the third harmonic of their repetition frequency, permitting TDS with a time delay resolution of better than $160 \mathrm{fs}$. Various beneficial aspects of the technique are demonstrated by performing pump-probe experiments on different samples with relevance for fundamental physics as well as for semiconductor metrology. Furthermore, a high-resolution THz-TDS system with exceptionally rapid data acquisition and greatly improved signal-to-noise ratio is realized.

\section{HIGH-SPEED ASOPS SYSTEM}

We employ a dual-laser system (Gigajet TWIN, Gigaoptics GmbH, Germany) comprising two Ti:sapphire femtosecond oscillators with repetition rates $f_{R, 1}$ (probe oscillator) and $f_{R, 2}$ (pump oscillator) of approximately $956 \mathrm{MHz}$ (i.e., the measurement window is $1046 \mathrm{ps}$ ). The oscillators are pumped by a diode-pumped solid-state laser at $532 \mathrm{~nm}$ wavelength (Verdi 6, Coherent Inc., Santa Clara, CA) whose $6.5 \mathrm{~W}$ output power is split into equal portions. The oscillators deliver approximately $600 \mathrm{~mW}$ of output power at a center wavelength tunable between 750 and $850 \mathrm{~nm}$ and a pulse duration of approximately $50 \mathrm{fs}$. The repetition rate difference $\Delta f_{R}=f_{R, 2}-f_{R, 1}$ is fixed using active feedback. The calibrated time delay between the two pulse trains is then given by $\tau(t)=\left(t-t_{0}\right) \times \Delta f_{R} / f_{R, 2}$. $t$ is the real-time elapsed after a trigger event used to start a measurement. $t_{0}$ is a static offset between a trigger event and the moment at which a pair of pulses from the two oscillators is coincident at the experiment location. The quality of the feedback electronics determines the experimental accuracy and stability of the true time-delay $\tau^{*}(t)$ as a function of real time versus the calibrated value $\tau(t)$. Residual timing jitter of the femtosecond oscillators directly determines this accuracy and stability and thereby the experimental time-delay resolution, especially if multiple traces are averaged. Instead of employing two feedback loops to stabilize the lasers to their individual reference synthesizers (sharing a common $10 \mathrm{MHz}$ clock) with an offset of $\Delta f_{R}$, ${ }^{1}$ here, the probe oscillator is used as master and the pump oscillator is linked to it as slave at a difference of $\Delta f_{R}=10 \mathrm{kHz}$ with a single feedback loop. ${ }^{6,7}$ The main advantage of such a scheme is that uncorrelated phase noise between the two individual synthesizers does not affect the time-delay resolution. Also, only the noise floor of one feedback loop enters the experiment. In contrast to previous work, ${ }^{6,7}$ the stabilization is performed at the third har- monic of the repetition frequency. This allows us to suppress imperfections of the stabilization electronics more efficiently than operating at the fundamental repetition rate.

The system setup is sketched in Fig. 1(a). A small portion of the probe oscillator's pulse train is detected with a fast Si-pin photodiode (PD1, model S7911, Hamamatsu Photonics, Japan) to obtain an electronic signal at $f_{R, 1}$. The bandwidth of PD1 is approximately $3 \mathrm{GHz}$ at a bias voltage of $15 \mathrm{~V}$, thus the signal additionally contains higher harmonics of the fundamental repetition frequency. The signal is split into a branch at the fundamental repetition rate and a branch at the third harmonic using a power splitter and bandpass filters at 1 and $3 \mathrm{GHz}$, respectively. The third harmonic signal is upshifted by $30 \mathrm{kHz}$ using a frequency shifter. See Fig. 2 for the upshifted signal at $3 \times f_{R, 1}$. The frequency shifter consists of a single sideband (SSB) generator with a design as described in Ref. 8 and an additional circuit that cancels leakage of the input signal (also referred to as carrier) through the SSB generator. To this end, a small portion of the SSB generator input is split off using a directional coupler and added to the output by means of a microwave combiner with the same amplitude but with a $180^{\circ}$ phase shift. This assembly allows us to suppress contributions at the carrier and the mirror-frequency $\left(f_{R, 1}-30 \mathrm{kHz}\right)$ by better than 50 $\mathrm{dB}$. However, apart from the carrier and mirror frequencies, additional sidebands at $30 \mathrm{kHz}$ and harmonics are still present on the signal due to nonlinearities within the frequency shifter. These cannot be fully eliminated. Their effect is discussed below. The pump oscillator's pulse train is detected with a second photodiode (PD2) whose signal is also split into a fundamental and a third harmonic branch. The third harmonic of $f_{R, 2}$ is phase locked to the upshifted third harmonic of $f_{R, 1}$. To this end, both signals are compared with a double-balanced mixer (DBM, model ZAM-42, Minicircuits Inc., Brooklyn, NY) serving as phase detector. The DBM output is supplied to a piezoelectric transducer (PZT) that supports a cavity mirror of the pump oscillator via a proportional-integral amplifier and a high-voltage amplifier. The time constant of the integral amplifier part has been set to $100 \mu \mathrm{s}$. The effective bandwidth of the entire feedback loop is $5 \mathrm{kHz}$ as has been characterized in an independent measurement. Here, this value is determined by the feedback bandwidth of the PZT mirror assembly. $\Delta f_{R}$ is now fixed to $10 \mathrm{kHz}$. Thus the time-delay window is scanned in $100 \mu \mathrm{s}$ of real time. The signals at $f_{R, 1}$ and $f_{R, 2}$ from the fundamental signal branches are supplied to a second DBM whose output is converted to a $10 \mathrm{kHz}$ transistor-transistor logic (TTL)level signal with less than $10 \mathrm{~ns}$ rise time. This signal can be used to trigger the data acquisition hardware. The TTL signal can be continuously delayed from 0 to $50 \mu$ s in order to adjust the timing between trigger event and the experimental data trace. We note that except for the frequency shifter and the DBMs, all electronic components used in the feedback loop and for trigger signal generation have been taken from a commercial repetition rate and timing stabilization unit (TL1000, Gigaoptics GmbH, Germany). 
(a)

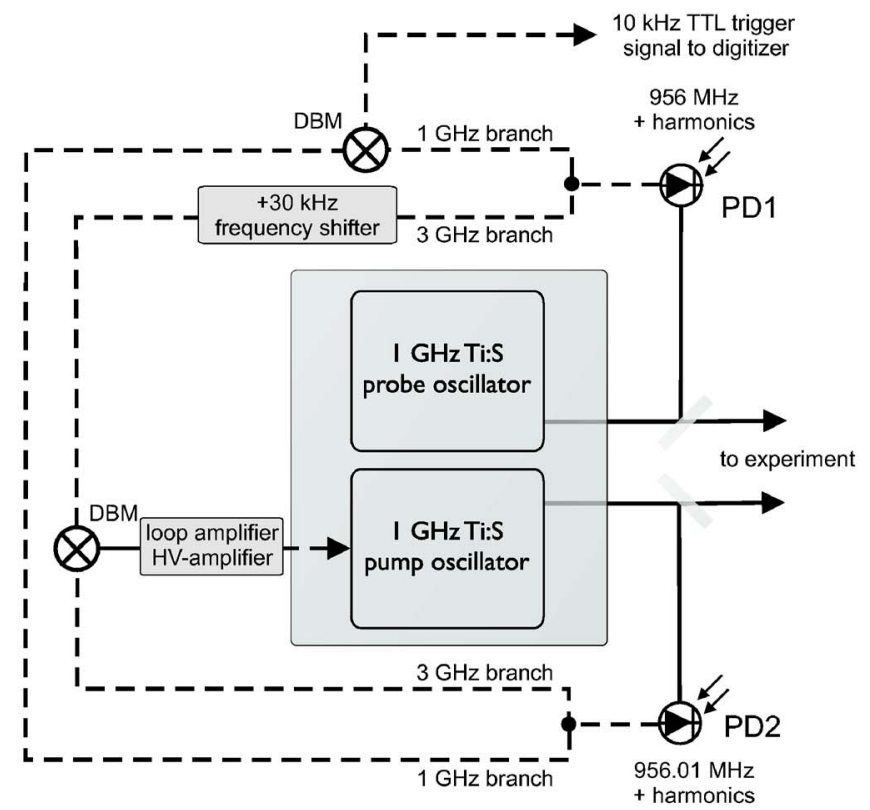

(b)
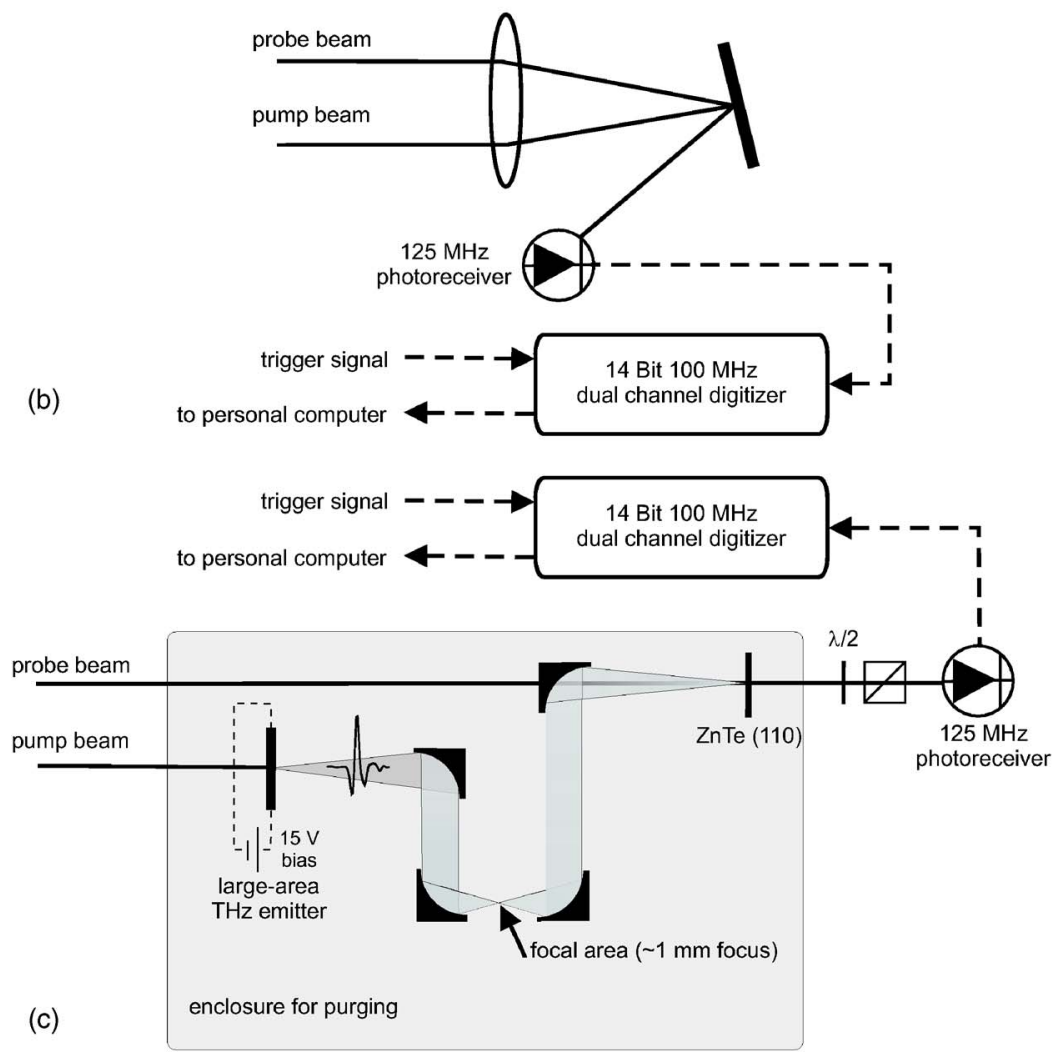

FIG. 1. (a) Sketch of high-speed ASOPS system. Optical paths are represented by solid lines, electrical signals by dashed lines. (b) Setup for optical pump-probe measurements. (c) High-speed ASOPS THz spectrometer setup.

\section{TIME-DELAY RESOLUTION AND CALIBRATION}

Apart from the laser pulse duration, the obtainable timedelay resolution $\Delta \tau$ of the high-speed ASOPS system is determined by several factors. In most cases, between $10^{2}$ and $10^{8}$ single traces are averaged for noise reduction. Limitations to the time-delay resolution due to the repetition rate stabilization can be expressed as $\Delta \tau_{S}=\sqrt{\left\langle\left(\tau_{i}^{*}(t)-\tau_{i}(t)\right)^{2}\right\rangle_{i}}$, i.e., the mean deviation of the true time-delay $\tau_{i}^{*}(t)$ from the calibrated time-delay $\tau(t)=\left(t-t_{0}\right) \times\left(\Delta f_{R} / f_{R, 2}\right)$, where the mean is taken over the total number $N$ of individual traces $i$. Residual timing jitter of the slave oscillator versus the reference signal due to limitations of the phase-locked loop (PLL) electronics is one source of such deviations. Of this residual jitter, only those contributions at Fourier frequencies higher than approximately $1 \mathrm{kHz}$ are significant. Timing jitter at Fourier frequencies lower than approximately one tenth of $\Delta f_{R}$ are not significant because the data acquisition system is triggered every $1 / \Delta f_{R}$, thus compensating for any lower frequency timing jitter.

An independent characterization of our PLL electronics revealed that the timing jitter of our femtosecond oscillators phase locked to an external reference signal amounts to approximately $95 \mathrm{fs}$ when integrated from 1 to $102 \mathrm{kHz}$ (jitter at higher Fourier frequencies is not significant at the given 


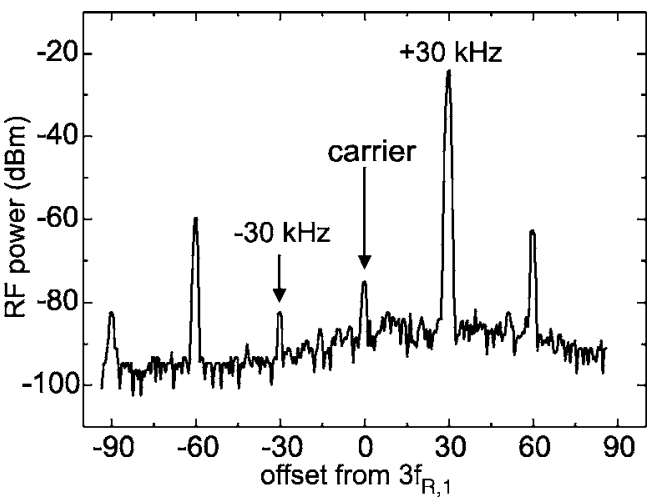

FIG. 2. (a) Frequency shifter output measured with a radio-frequency spectrum analyzer. The main peak at $f_{R, 1}+30 \mathrm{kHz}$ is used as reference for the save oscillator feedback loop. Modulations at harmonics of $30 \mathrm{kHz}$ are residual carrier and mirror frequency signals and contributions due to nonlinearities in the frequency shifter.

level of total jitter). This gives an estimate of the influence of the PLL electronics on $\Delta \tau_{S}$. Another source for deviations of the $\tau_{i}^{*}(t)$ from $\tau(t)$ are imperfections of the frequency shifter that are imposed on the slave oscillator via the feedback loop in addition to the PLL noise floor. The frequency shifter adds broadband noise as well as sidebands at harmonics of $30 \mathrm{kHz}$ to the shifted $f_{R, 1}$ signal used as reference for $f_{R, 2}$ (see Fig. 2 ). The broadband noise creates additional timing jitter similar to the noise floor of the PLL electronics and thus further increases $\Delta \tau_{S}$. Generally, $\Delta \tau_{S}$ is a function of $t$ and increases linearly with the real time elapsed after a trigger event during which the experiment accumulates timing jitter until the next trigger event occurs. Thus $\Delta \tau$ depends on the position $\tau(t)$ within the measurement window and should be written as $\Delta \tau(\tau)$. The sidebands created by the frequency shifter appear as parasitic $30 \mathrm{kHz}$ modulations on the error signal that controls the slave oscillator repetition rate via the PZT. These modulations create a periodic timing error in phase with the $10 \mathrm{kHz}$ trigger signal. This timing error causes systematic deviations of the true time-delay $\tau_{i}^{*}(t)$ with respect to the calibrated time-delay $\tau(t)=\left(t-t_{0}\right) \times\left(\Delta f_{R} / f_{R, 2}\right)$. This effect is the main reason to perform the stabilization at a higher repetition frequency harmonic. Operating at the fundamental frequency would result in sidebands at $10 \mathrm{kHz}$. These would be closer to the required feedback loop bandwidth of $5 \mathrm{kHz}$ and thus converted much more efficiently to systematic timing errors.

The final limitation to the overall time-delay resolution $\Delta \tau$ in a high-speed ASOPS experiment is given by the available bandwidth $B$ to detect the probe beam and digitize the signal. In the experiments presented here, the probe beam is detected using a $125-\mathrm{MHz}$ bandwidth photoreceiver. A 14-bit digitizer with a bandwidth of $100 \mathrm{MHz}$ [or optional 200 MHz using two interleaved $100 \mathrm{MHz}$ analog-to-digital (A/D) converters] is employed to digitize the data. The effective $B$ is thus $100 \mathrm{MHz}$, yielding 10000 data points for each $100 \mu$ s measurement cycle. With an available time-delay window of $1 \mathrm{~ns}$, this results in one data point for every $100 \mathrm{fs}$ of time delay. This value represents the lower limit to the overall time-delay resolution $\Delta \tau$ regardless of $\Delta \tau_{S}$ given by the repetition frequency offset stabilization. For a given rep-

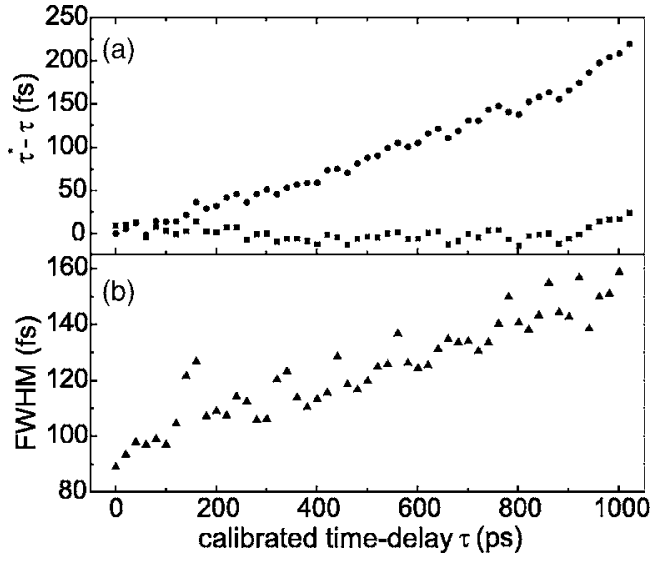

FIG. 3. (a) Circles: Deviation of the true time delay from the calibrated time delay vs calibrated time delay. Squares: Same data as circle symbols with linear contribution due to residual stepper motor misalignment subtracted. (b) FWHM of second cross-correlation peak vs calibrated time delay.

etition rate and offset frequency, the time-delay resolution limit due to the available acquisition bandwidth $B$ can be written as $\Delta \tau_{D}=\left(\Delta f_{R} / f_{R, 2}\right) \times B^{-1}$. Thus if the same $\Delta \tau_{D}$ was to be achieved with standard femtosecond oscillators with $100 \mathrm{MHz}$ repetition frequency, $\Delta f_{R}$ would have to be reduced to $1 \mathrm{kHz}$. This lower value, however, has lead to a time-delay resolution of worse than approximately $2 \mathrm{ps}$ in our experiments for two reasons. First, a reduced $\Delta f_{R}$ increases the time between two trigger events during which the experiment accumulates timing jitter. Second, parasitic signals occurring at $\Delta f_{R}$ within the feedback electronics lie well within the required bandwidth of the repetition rate control loop and significantly interfere with the stabilization.

The significance of the above mentioned effects within our system has been tested in order to assess their experimental impact. To this end, the true time-delay $\tau^{*}$ and the overall time-delay resolution $\Delta \tau$ have been characterized as function of position $\tau$ within the measurement window. These data were extracted from a cross-correlation signal between a double-pump pulse and a probe pulse generated in a BBO crystal. Here, the $200 \mathrm{MHz}$ sampling rate option of our digitizer has been employed such that the effective $B$ has been $125 \mathrm{MHz}$, given by the photoreceiver. The doublepump pulse was created using a Michelson interferometer where the length of one arm and thus the true time delay between the pump pulses was adjustable via a stepper motor with $100 \mathrm{~nm}$ resolution. The first pump pulse was more intense than the second such that the first cross-correlation peak could be used to trigger the data acquisition using the rising signal edge $\left(t_{0}\right.$ is well below the laser pulse duration and thus negligible). The second peak was used for characterization purposes. In this experiment, $\tau^{*}$ is given as the delay between the pump pulses as a result of the path length difference $D$, i.e., $\tau^{*}=2 D / c . t$ is the real-time that elapses between the two cross-correlation peaks as measured with the data acquisition hardware and is used to calculate $\tau(t)$. For each measurement at a different value of $\tau, 10.000$ traces were averaged, i.e., the acquisition time had been set to approximately $1 \mathrm{~s}$.

The square symbols in Fig. 3(a) represent the deviation 
of the true $\tau^{*}(t)$ relation from the calibrated value $\tau(t)$ $=\left(t-t_{0}\right) \times\left(\Delta f_{R} / f_{R, 2}\right)$ as a function of $\tau$. A dominant linear contribution of approximately 200 fs per available timedelay window of $f_{R, 2}^{-1}$ is observed. This linear deviation does not originate from the stabilization scheme but stems from a residual misalignment of the stepper motor. This was verified by repeating the same measurement with two fundamentally different stabilization methods: (i) using a frequency shifter at the fundamental repetition rate and (ii) using two individual $\mathrm{GHz}$ synthesizers as references. ${ }^{1,6,7}$ In addition, different values of $\Delta f_{R}(5$ and $15 \mathrm{kHz})$ were employed. In all cases a linear deviation with the same slope was observed, confirming that the linear contribution is very likely not caused by the repetition frequency stabilization. We thus subtract the linear contribution from the measured data in order to arrive at the contribution from the stabilization electronics as shown in Fig. 3(a). The data show a maximum deviation of approximately $25 \mathrm{fs}$, which is insignificant compared to the time-delay resolution of the system. Thus, the imperfections of the frequency shifter do not cause a significant error in the time-delay axis calibration.

Figure 3(b) shows the full width at half-maximum of the second cross-correlation peak as a function of calibrated $\tau$. At the beginning of the measurement window the time-delay resolution is $89 \mathrm{fs}$, given by the available detection bandwidth $B$ (the theoretical value is $84 \mathrm{fs}$ ). At later positions in the measurement window, the time-delay resolution degrades linearly and reaches a maximum of 159 fs. This value represents the combined effect of the PLL electronics noise floor and the broadband noise added to the reference signal by the frequency shifter. Thus, our setup is capable of performing ultrafast spectroscopy over a $1 \mathrm{~ns}$ time-delay window with a time-delay resolution of better than $160 \mathrm{fs}$.

\section{PUMP-PROBE SPECTROSCOPY WITHOUT MECHANICAL DELAY LINE}

High-speed ASOPS is particularly useful for the investigation of transient phenomena that require a combination of femtosecond time-delay resolution and access to a timedelay window of several hundreds of picoseconds. Here, we discuss various beneficial aspects of the technique for pumpprobe spectroscopy using two different samples. The setup is shown in Fig. 1(b). The pump and probe oscillator beams, both with a central wavelength of $800 \mathrm{~nm}$, are focused onto the sample with a spot size of approximately $30 \mu \mathrm{m}$. The reflected probe beam is adjusted to $5 \mathrm{~mW}$ and detected with the $125 \mathrm{MHz}$ photoreceiver whose output is recorded with the digitizer set to the single A/D converter mode with a $100 \mathrm{MHz}$ sampling rate, i.e., $\Delta \tau_{D}=105$ fs. The $200 \mathrm{MHz}$ mode with two interleaved $100 \mathrm{MHz}$ A/D converters is not useful for the digitization of small signals because the A/D converters exhibit an uncontrollable and temporally varying offset. For these measurements, we have used the electronically generated $10 \mathrm{kHz}$ TTL signal to trigger the data acquisition. This is favorable because a small part of the signal is usually lost if signal triggering is employed.

Coherent phonon spectroscopy has been an important field for the investigation of fundamental energy relaxation
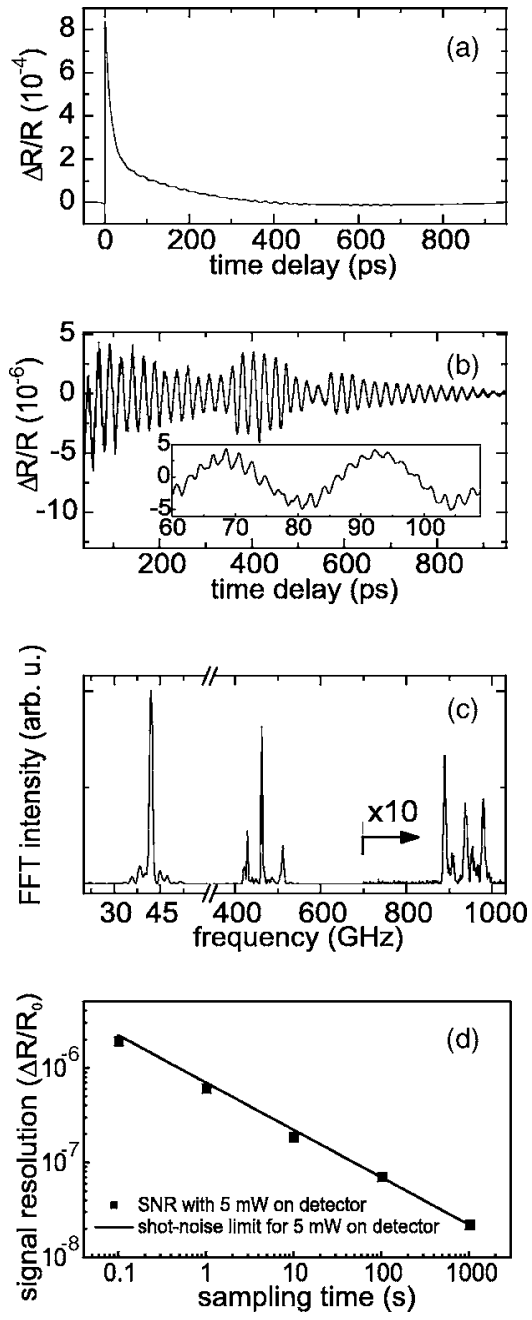

FIG. 4. (a) Reflectivity changes of $(\mathrm{GaAs})_{19}(\mathrm{AlAs})_{19}$ superlattice after impulsive optical excitation. (b) Extracted oscillatory contributions due to coherent acoustic phonons (inset shows zoom into early time delays revealing details of contributions due to zone-folded acoustic phonons). (c) Fouriertransform of data shown. (b) Data are scaled by a factor 10 above $700 \mathrm{GHz}$. (d) Signal resolution of pump-probe setup vs total measurement time (square symbols) and shot-noise limit (solid line).

processes in semiconductor and semiconductor heterostructures in the past. $^{9}$ As a typical example, we have chosen a $(\mathrm{GaAs})_{19}(\mathrm{AlAs})_{19}$ superlattice with 40 periods. The response of such structures to impulsive optical excitation is well known from earlier experiments performed with conventional pump-probe spectroscopy systems: Coherent longitudinal acoustic phonons with periods between 20 and 1 ps and lifetimes exceeding $100 \mathrm{ps}$ are observable through weak transient reflectivity changes superimposed on a strong exponentially decaying carrier relaxation background. Figure 4(a) shows the reflectivity changes induced by the impulsive generation of electron-hole pairs in the superlattice at room temperature. The pump and probe oscillators were resonant with the first electron-hole interband transition of the superlattice at $800 \mathrm{~nm}$. The acquisition time was $11 \mathrm{~s}$. The initial rise due to the interband excitation decays through recombination and diffusion of carriers and is on the order of $10^{-4}$. Superimposed is an oscillating contribution originating from coherent longitudinal acoustic phonons with an amplitude on the order of $10^{-6}$. This signature is extracted by subtracting 


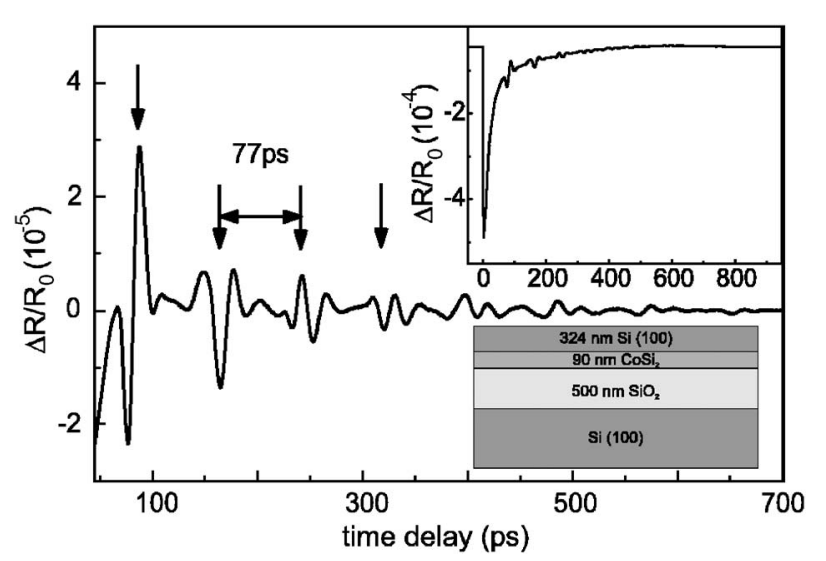

FIG. 5. Extracted modulations of raw data (shown in top inset) due to multiple reflections of an acoustic pulse within the top Si layer of a siliconon-metal-on-insulator structure (bottom inset).

the electronic background as an exponential decay [see Fig. 4(b)]. The Fourier spectrum of the phonons is shown in Fig. 4(c). It reveals a strong mode close to $40 \mathrm{GHz}$, which corresponds to coherent phonons on the Brillouin branch of the longitudinal acoustic dispersion excited with twice the light wave vector. The linewidth is $1.2 \mathrm{GHz}$ which presumably represents the resolution limit of the particular measurement. This limit is expected to be marginally greater than $1 \mathrm{GHz}$ due to the fact that $t_{0}$ was approximately $80 \mathrm{ps}$ and thus the measured time-delay window after excitation only 960 ps. Additionally, first and second order back-folded modes are clearly resolved in the spectrum at approximately 480 and $960 \mathrm{GHz}$, respectively. These higher frequencies correspond well with the calculated phonon dispersion. ${ }^{10}$ They appear as triplets, since the detection process selects the modes at zero wave vector and those at twice the wave vector of the probe light. Figure 4(d) shows the signal resolution of data taken at different acquisition times which is in excellent agreement with the shot-noise limit for $5 \mathrm{~mW}$ incident on the detector. These data demonstrate the capability of high-speed ASOPS to observe combinations of fast and slow dynamics with high sensitivity within only a few seconds.

As a second example, we have chosen the field of laser based ultrasound as an application with relevance for semiconductor metrology. ${ }^{11,12}$ Here, the transit times of laser induced acoustic pulses are measured in order to determine thicknesses within multilayer systems in a noninvasive way. Using femtosecond pulses in conjunction with appropriate models for the acoustic pulse propagation, a depth resolution in the subnanometer range can be obtained. ${ }^{13}$ The typical required observation windows are in the range of hundreds of picoseconds. For example, a single Si layer of $1 \mu \mathrm{m}$ thickness exhibits a transit time of an acoustic pulse of $118 \mathrm{ps,} \mathrm{a}$ time scale for which high-speed ASOPS is well-matched. The inset of Fig. 5 shows transient reflectivity changes of a $324 \mathrm{~nm}$ Si layer wafer bonded onto a $\mathrm{CoSi}_{2}$ layer after impulsive excitation. The whole structure of a silicon-on-metalon-insulator consists of a top $\mathrm{Si}$ layer, a buried $\mathrm{CoSi}_{2}$ layer, and a buried $\mathrm{SiO}_{2}$ layer on a $\mathrm{Si}$ (100) substrate which is formed using Co silicidation, wafer bonding, and wafer splitting (see inset of Fig. 5). Such substrates are employed in a
Bipolar Complementary Metal Oxide Semiconductor (BiCMOS) process. ${ }^{14}$ Figure 5 shows the modulations due to multiple reflected acoustic pulses within the top layer extracted from the exponentially decaying thermal background. The reflections are equally spaced and exhibit characteristic sign changes after each reflection from a Si-air interface. The roundtrip time within the $\mathrm{Si}$ layer is $77 \mathrm{ps}$. With the longitudinal sound velocity of $8.43 \times 10^{5} \mathrm{~cm} / \mathrm{s}$ for the (001) direction of silicon, this corresponds to a layer thickness of 324 $\mathrm{nm}$ in excellent agreement with TEM results on this sample. The acquisition time required for this measurement was $1 \mathrm{~s}$. This example demonstrates that high-speed ASOPS enables thickness metrology based on laser-induced ultrasound with measurement speeds unobtainable with mechanical delay devices.

\section{TERAHERTZ TIME-DOMAIN SPECTROSCOPY AT $10 \mathrm{kHz}$ SCAN RATE WITH $1 \mathrm{GHz}$ RESOLUTION}

terahertz spectroscopy is a rapidly evolving field due to its relevance for the characterization and identification of a wide variety of materials, e.g., through measurements of absorption or reflectivity spectra. ${ }^{15,16}$ THz-TDS is particularly advantageous because of the large possible bandwidth on the order of several THz. Also, unlike for most continuous-wave methods, cryogenically cooled detectors are not required and supplies of liquid nitrogen and helium are not necessary. Many possible applications of THz-TDS are yet hampered by the slow scanning speeds of conventional mechanical delay generators. Examples are the screening of mail or other opaquely packaged items for explosives, ${ }^{2}$ hazardous substances, or illicit drugs, the readout of DNA binding state sensors ${ }^{1}$ or similar monitoring tasks requiring a high throughput. Furthermore, applications where experimental conditions only exist for short times or where dynamic phenomena are to be investigated on a millisecond time scale would greatly benefit from faster time-delay scanning speeds (e.g., experiments in pulsed high-magnetic fields, reaction dynamics in biological samples). Finally, a reduction of the scan time per pixel would be advantageous for imaging applications of THz-TDS. High-speed ASOPS thus bears great potential to advance the field of THz-TDS in general.

Here, we demonstrate a high-speed ASOPS based THz time domain spectrometer and its application to transmission spectroscopy of water vapor as a well-characterized model substance. The setup is shown in Fig. 1(c). The pump oscillator drives a $\mathrm{THz}$ emitter device which is composed of a large-area interdigitated finger structure with an electrode spacing of $5 \mu \mathrm{m}$ on a semi-insulating GaAs substrate $\mathrm{e}^{17,18}$ (Tera-SED, Gigaoptics GmbH, Germany). Optical excitation is performed under normal incidence with an average power of $600 \mathrm{~mW}$. The device is dc biased at $15 \mathrm{~V}$ which results in an emission bandwidth of approximately $2.5 \mathrm{THz}$ at $10 \mathrm{~dB}$ below the maximum. The photocurrent through the emitter is $50 \mathrm{~mA}$. The advantage of this emitter in comparison to photoconductive antennas with small electrode gap spacing is the possibility to dissipate a greater excitation power. ${ }^{17,18}$ The $\mathrm{THz}$ pulse is emitted through the GaAs substrate and collected with a $90^{\circ}$ off-axis parabolic mirror with $5 \mathrm{~cm}$ focal length. It is focused with another off-axis parabolic mirror to 
a spot of approximately $1 \mathrm{~mm}$ diameter. The THz pulse is again collimated and focused for detection based on the electro-optic effect in a (110) ZnTe crystal of $600 \mu \mathrm{m}$ thickness. ${ }^{19,20}$ The optical probe beam is focused to the same spot as the $\mathrm{THz}$ pulse on the detector crystal through a hole with $2 \mathrm{~mm}$ diameter in an off-axis parabolic mirror. This arrangement does not block or modify most of the $\mathrm{THz}$ radiation. Experiments are performed at a probe wavelength of $820 \mathrm{~nm}$. The probe beam polarization behind the ZnTe crystal is analyzed with a half-wave plate $(\lambda / 2)$ and a polarizing beam splitter (PBS). Instead of using the common scheme where the difference between perpendicular polarizations is detected, we place a single photoreceiver in one of the beams behind the beam splitter to obtain the electro-optic signal. Compared to the conventional approach, we lose a factor of two in signal amplitude but circumvent bandwidth limitations introduced by available difference amplifiers.

A $\mathrm{THz}$ transient acquired under nitrogen purging within $1.1 \mathrm{~s}$ of acquisition time is shown in Fig. 6(a). Only the first 80 ps of the full $1 \mathrm{~ns}$ data are plotted for better visibility. The echoes appearing at a spacing of approximately $16 \mathrm{ps}$ are due to multiple reflections within the $700 \mu \mathrm{m}$ thick emitter substrate. No visible noise is present on the trace at negative time delays. A Fourier-transform power spectrum of the data [see Fig. 6(b)] reveals a signal-to-noise ratio of $\approx 37 \mathrm{~dB}$. A more detailed data analysis shows that the signal-to-noise ratio is in fact shot-noise limited, i.e., it increases linearly with data acquisition time. Also shown in Fig. 6(b) is a Fourier-transform spectrum of data acquired with water vapor present in the setup within $110 \mathrm{~s}$ of acquisition time. The signal-to-noise ratio amounts to $57 \mathrm{~dB}$. Using those data and a reference spectrum acquired with the same acquisition time and signal-to-noise ratio under nitrogen purging (not shown), we calculated a water vapor transmission spectrum as shown in the lower panel of Fig. 6(c). Also shown in Fig. 6(c) is the dynamic range limit given by the signal-to-noise ratio of the reference spectrum. A zoom into the pressure broadened line at approximately $1.4 \mathrm{THz}$ (with a linewidth of approximately $10 \mathrm{GHz}$ ) endorses that our system yields one data point per gigahertz (see inset). The upper panel of Fig. 6(c) shows a transmission spectrum calculated for our experimental conditions using data from the HITRAN database on an inverted scale. ${ }^{21}$ The agreement between our measurement and the HITRAN data is good for frequencies up to $2.5 \mathrm{THz}$. Differences between the line frequencies are below our resolution limit. Differences in the transmission at values less than approximately $10^{-3}$ are attributed to residual water content in our setup under nitrogen purging and to the limited dynamic range at higher frequencies.

In comparison to our earlier work, ${ }^{7}$ the signal-to-noise ratio of the presented system has been increased by approximately $20 \mathrm{~dB}$ by means of several improvements to the system (increased probe power on the photoreceiver and decreased $\mathrm{THz}$ radiation losses). Furthermore, the sensitivity at frequencies greater than $2.5 \mathrm{THz}$ was increased by about $3-5 \mathrm{~dB}$ due to the improved time-delay resolution of the system.
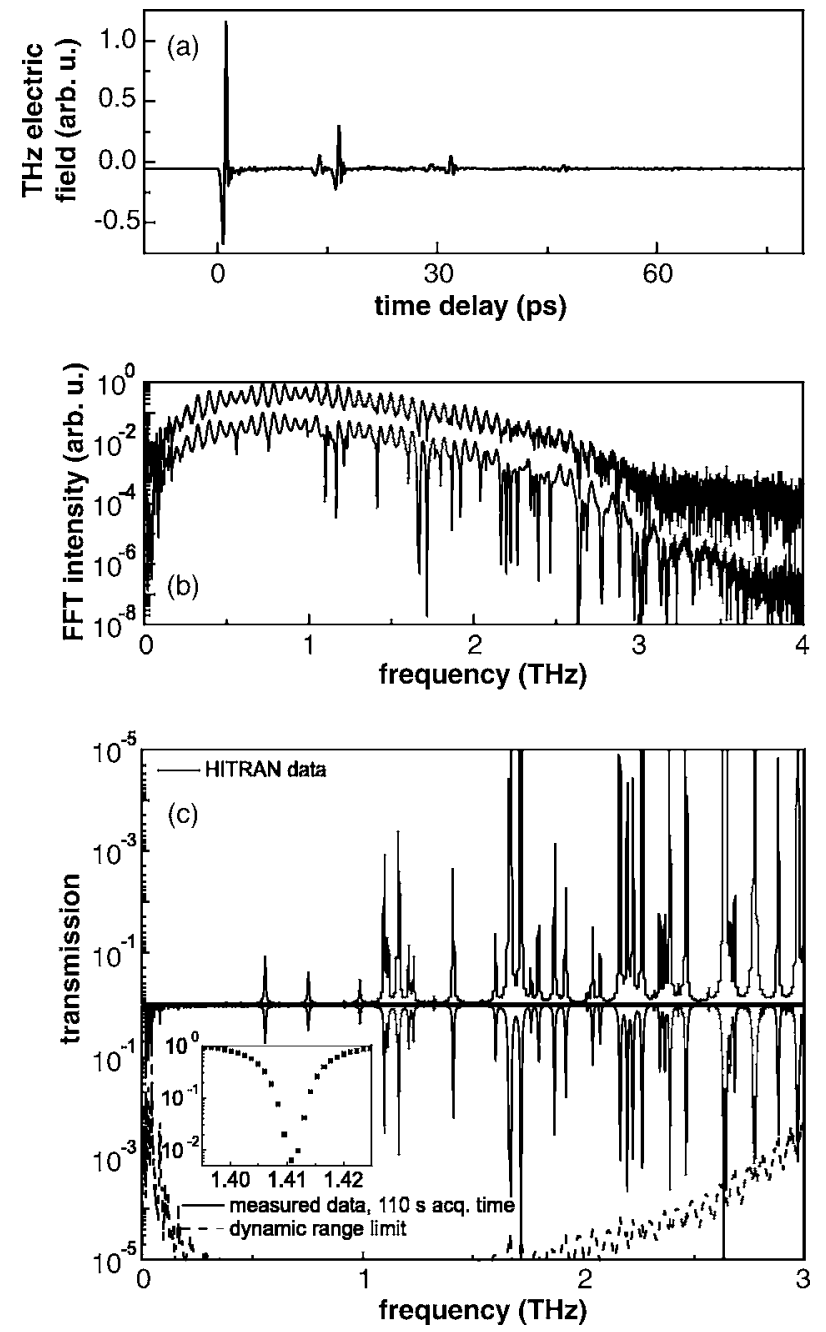

FIG. 6. (a) THz transient acquired within $1 \mathrm{~s}$ acquisition time in a nitrogen purged setup. (b) Fourier-transform spectrum of data shown in panel (a) (top curve) and of data acquired within $110 \mathrm{~s}$ acquisition time with water vapor present in the setup (bottom curve). Curves are offset one order of magnitude for better visibility. (c) Top panel: water vapor transmission spectrum for our experimental conditions compiled from the HITRAN database. Bottom panel: measured water vapor transmission spectrum (solid line) together with the frequency dependent dynamic range limit of the setup (dashed line, data are partly outside plotted range). The inset shows a zoom into the line at approximately $1.4 \mathrm{THz}$.

\section{CONCLUSION}

A high-speed ASOPS time-domain spectroscopy system based on two femtosecond Ti:sapphire femtosecond oscillators with pulse repetition frequencies close to $1 \mathrm{GHz}$ has been demonstrated. The oscillator's pulse repetition frequencies are locked at their third harmonic with a $10 \mathrm{kHz}$ offset using a master-slave configuration. With this scheme the relative time delay between the emitted pulse trains is scanned over a time-delay window of $1 \mathrm{~ns}$ at a rate of $10 \mathrm{kHz}$. Such a combination of rapid scanning and long time-delay window ( $1 \mathrm{~ns}$ is equivalent to $30 \mathrm{~cm}$ path length variation) is impossible with conventional schemes employing mechanical time-delaying devices. A detailed characterization has shown that the system maintains a time-delay resolution of better than $160 \mathrm{fs}$ when multiple traces are averaged for noise reduction. The presented high-speed ASOPS apparatus has been employed for optical pump-probe spec- 
troscopy where transient reflectivity changes of an impulsively excited sample can be measured with a relative signal resolution of $6 \times 10^{-7}$ within just $1 \mathrm{~s}$ of acquisition time. As an example, coherent phonons in a semiconductor superlattice have been detected with a spectral resolution of $1.2 \mathrm{GHz}$. Furthermore, we have demonstrated the feasibility of performing highly accurate semiconductor layer thickness metrology by measuring the $324 \mathrm{~nm}$ thickness of the $\mathrm{Si}$ top layer of a multilayer BiCMOS structure within just $1 \mathrm{~s}$ of acquisition time. Such performance may be very useful for high-throughput in situ screening of layered materials. In the field of THz-TDS, our system allows us to perform transmission spectroscopy with a bandwidth exceeding $3 \mathrm{THz}$ and a spectral resolution of $1 \mathrm{GHz}$. THz time-domain spectra with signal-to-noise ratios of 37 and $57 \mathrm{~dB}$ are obtained within 1 and $110 \mathrm{~s}$ of acquisition time, respectively. This performance is believed to have enormous impact on the capabilities to conduct screening applications in the $\mathrm{THz}$ frequency range at a high throughput.

\section{ACKNOWLEDGMENTS}

The authors are grateful to H. Harde for providing transmission data compiled from the HITRAN database shown in Fig. 6. The authors thank K. Köhler, M. Wiemer, and S. Mantl for providing samples used for the presented experiments. This work has been partially supported by the Deutsche Forschungsgemeinschaft (DE456/9) and the Ministry of Science, Research and the Arts of Baden-Württemberg.

${ }^{1}$ C. Janke, M. Först, M. Nagel, H. Kurz, and A. Bartels, Opt. Lett. 30, 2357 (2005).

${ }^{2}$ Y. C. Shen, T. Lo, P. F. Taday, B. E. Cole, W. R. Tribe, and M. C. Kemp,
Appl. Phys. Lett. 86, 241116 (2005).

${ }^{3}$ P. A. Elzinga, F. E. Lytle, Y. Jian, G. B. King, and N. M. Laurendeau, Appl. Opt. 26, 4303 (1987).

${ }^{4}$ T. Yasui, E. Saneyoshi, and T. Araki, Appl. Phys. Lett. 87, 061101 (2005).

${ }^{5}$ M. S. Brown, G. J. Fiechtner, J. V. Rudd, D. A. Zimdars, M. Warmuth, and J. R. Gord, Appl. Spectrosc. 60, 261 (2006).

${ }^{6}$ A. Bartels, F. Hudert, C. Janke, T. Dekorsy, and K. Köhler, Appl. Phys. Lett. 88, 041117 (2006).

${ }^{7}$ A. Bartels, A. Thoma, C. Janke, T. Dekorsy, A. Dreyhaupt, S. Winnerl, and M. Helm, Opt. Express 14, 430 (2006).

${ }^{8}$ K. W. Holman, Dissertation, Appendix B, University of Colorado (2005), published online at http://jilawww.colorado.edu/pubs/thesis/holman/ holman_thesis.pdf.

${ }^{9}$ T. Dekorsy, G. Cho, and H. Kurz, in Light Scattering in Solids VIII, Topics in Applied Physics 76, edited by M. Cardona and G. Güntherodt (Springer, Berlin, 2000), pp. 169-209.

${ }^{10}$ T. Dekorsy, F. Hudert, R. Cerna, H. Schäfer, C. Janke, A. Bartels, K. Köhler, S. Braun, M. Wiemer, and S. Mantl, Proc. SPIE 6393, 63930H-1 (2006).

${ }^{11}$ H. Maris, Sci. Am. 1, 64 (1998).

${ }^{12}$ H. Y. Hao, H. J. Maris, and D. K. Sadana, Electrochem. Solid-State Lett. 54, 1 (1998).

${ }^{13}$ See, $\quad$ e.g., http://www.rudolphtech.com/pdfs/products/ PULSE_Technology.pdf

${ }^{14}$ Q. T. Zhao, H. L. Bay, S. Zimmermann, M. Wiemer, C. Kaufmann, B. Trui, H. Höhnemann, V. Dudek, and S. Mantl, Semicond. Sci. Technol. 21, 157 (2006).

${ }^{15}$ H. Harde and D. Grischkowsky, J. Opt. Soc. Am. B 8, 6038 (2001).

${ }^{16}$ Sensing with $\mathrm{THz}$ Radiation, edited by D. Mittleman (Springer, Heidelberg, 2002) and references therein.

${ }^{17}$ A. Dreyhaupt, S. Winnerl, T. Dekorsy, and M. Helm, Appl. Phys. Lett. 86, 121114 (2005).

${ }^{18}$ A. Dreyhaupt, S. Winnerl, M. Helm, and T. Dekorsy, Opt. Lett. 31, 1546 (2006).

${ }^{19}$ Q. Wu and X.-C. Zhang, Appl. Phys. Lett. 68, 1604 (1996).

${ }^{20}$ R. Huber, A. Brodschelm, F. Tauser, and A. Leitenstorfer, Appl. Phys. Lett. 76, 3191 (2000).

${ }^{21}$ High-resolution transmission molecular absorption database (HITRAN), http://cfa-www.harvard.edu/HITRAN/ 